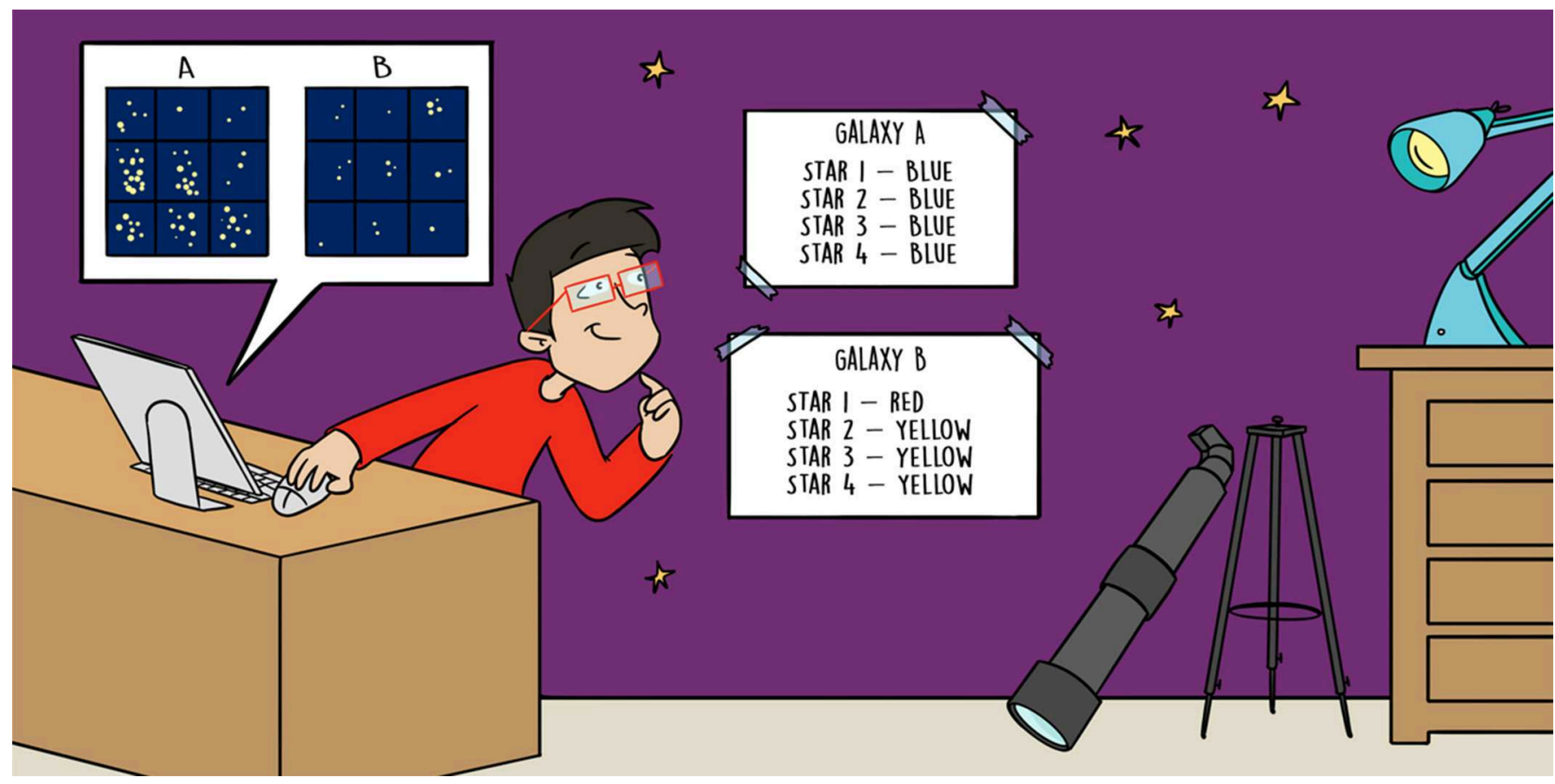

\title{
MEASURING DISTANCES TO GALAXIES
}

\section{Jonathan D. Davis *}

The Physics Department, Utah Valley University, Orem, UT, United States

YOUNG REVIEWERS:

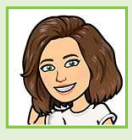

\section{CAYLE}

AGE: 13

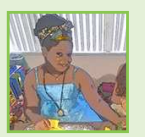

EILEEN

AGE: 8

\section{ALPHA CENTAURI}

The closest star to our solar system. It is 1.37 parsecs, or 41.53 trillion kilometers, from Earth.
Measuring distances to other galaxies is an important part of our ability to understand how the universe works. Astronomers can use what are called surface brightness fluctuations (SBF, for short), along with the color of a galaxy, to calculate how far away it is from earth. Most galaxies measured in this way are millions of light years away.

\section{SPACE IS BIG}

In his book, The Hitchhikers Guide to the Galaxy, Douglas Adams says, "Space is big. Space is really big! You just would not believe how vastly hugely mindbogglingly big it is [1]."

He was not kidding either! Imagine it this way. Pretend you are on the Autobahn in Germany. You are in a Ferrari, zooming at 300 kph (186 mph). If you did not have to stop, you could go around the earth in about $133 \mathrm{~h}$, or 5 and a half days. Now imagine if your Ferrari suddenly could travel to the sun. It would take nearly 4,000 times longer to get to the sun than to drive around the earth! That is $\sim 22,000$ days in the Ferrari! The closest star that is not the sun is called Alpha Centauri. To 
1 I got all my numbers from this website: https://www. wolframalpha.com/. Just type in the name of a star or galaxy you want to know more about.

\section{GALAXY}

A bunch of stars, maybe even trillions that all clump together and are in orbit around each other.

\section{ANDROMEDA}

GALAXY

One of the closest galaxies to our own galaxy, the Milky Way.

\section{PARSEC}

A way that astronomers describe distances in space. One parsec is the same as 30.86 trillion kilometers.

\section{SURFACE}

BRIGHTNESS

FLUCTUATIONS

(SBF)

How bumpy light appears in a picture of a galaxy from place to place. It is what we measure to help determine a galaxy's distance.

PIXEL

A very small light that is a single part of a screen. A TV is made up of many pixels together get there, it would be like driving to the sun almost 300,000 times! The closest galaxy, Andromeda, is nearly 600,000 times farther than Alpha Centauri! The farthest galaxies I measure are over 100 times farther than Andromeda, and to get to the end of the visible universe, you have to go almost 150 times farther than that! ${ }^{1}$ Anyway, if you wanted to drive to the end of the visible universe, or pretty much anywhere out in space, it would take a really long time.

\section{Measuring Space With Parsecs}

Because space is so big, many astronomers do not like to say how far away things are using miles or kilometers. Instead, we use a measurement that we call a parsec. Remember Alpha Centauri, the closest star? It is 1.347 parsecs, or 41,560,000,000,000 (or 41.56 trillion) kilometers away. I like parsecs because, for me, they are easier to use and understand compared with all the zeros there are when we use kilometers.

\section{BUMPY GALAXIES}

It is pretty cool that space is so huge, or at least I think so. But how do we even know how big space is? There are many different ways to measure things in space, but I focus on measuring distance to galaxies using a very special method called surface brightness fluctuations (SBF). To explain how SBF works, take a close look at the phone or computer screen you are reading this on. If the screen is really close to your face, you will be able to see the pixels, or the tiny lights that make up the image you see, on the screen. Now, back away until you are no longer able to see the pixels.

You can make out pixels on your screen when it is close because your screen is just made up of a lot of pixels. Similarly, galaxies are just a bunch of stars clumped together. Now a galaxy is not a phone screen but it does behave in a similar way. When galaxies are close to us, we see bigger bumps coming from that galaxy due to how the galaxies stars are organized. Just like a screen, when galaxies are farther away, all those stars blend together, and the galaxy will look really smooth, similar to the way the pixels on a screen blend together when you sit back from it. In Figure 1, you can see how a closer galaxy looks bumpier than one that is farther away. When we know the size of the bumps, because of the way stars are organized in a galaxy, it helps astronomers figure out how many stars that galaxy has.

\section{Measuring Bumps}

To measure the size of the "bumps" in a far-away galaxy, we need to remove the main part of the galaxy from the picture to focus on the bumps. To do this, a computer creates an image of a really smooth-looking galaxy that resembles the one we have taken a picture of. We then take the smooth galaxy image which was generated by 
Figure 1

The picture on the left is the galaxy M32, which is right next to the Andromeda galaxy and is 0.77

megaparsecs (Mpc), or 770,000 parsecs, away The galaxy on the right is NGC 7768. It is 120 Mpc, or $120,000,000$ parsecs, away. M32 looks much bumpier than NGC 7768 because it is closer to us. Both pictures come from the Gemini observatory $^{2}$.

\section{POWER SPECTRUM}

This gives information about the sizes of all the bumps we see in a picture of a galaxy.

2 http://www.gemini. edu/ is a website where you can learn more about the Gemini telescope.

\section{GALAXY COLOR}

The color of a galaxy tells us how hot the stars in that galaxy are.

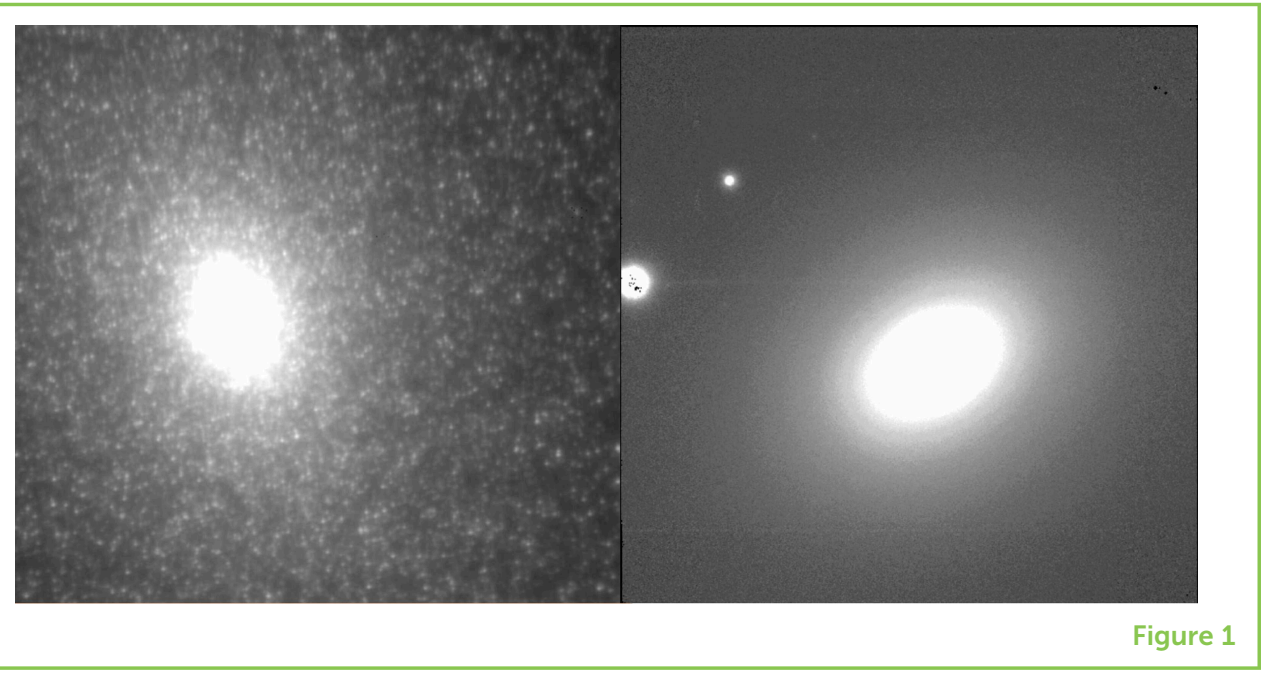

the computer and subtract it from the image in our original picture, leaving only the galaxy's bumps as shown in Figure 2. Once we have only the bumps in our picture, we next need to figure out something called the power spectrum. The power spectrum tells us how many big bumps a galaxy has, compared with how many small bumps it has. If the power spectrum tells us that there are a lot of detailed, sharp bumps in the image, that could mean the galaxy is closer to us. If the same galaxy is farther away, the power spectrum will show only less detailed, smoother bumps in the image. If you know the color of that galaxy and how many stars it has, you can then figure out how much light the you should see if it is a certain distance away.

\section{COLORFUL GALAXIES}

Imagine you are with your friends sitting around a campfire, roasting some delicious marshmallows. Have you ever wondered why some parts of the fire are red, some are orange, some are yellow, and some white? This is because cooler parts of the fire look red, hotter parts look orange, even hotter parts look yellow, and the hottest are white. If you could get the fire hot enough, it would even start to look blue (but it would also give you a very bad sunburn). Galaxies are the same way. Just like fire, when galaxies contain colder stars, they look red. When they contain hotter stars, they look bluer. When we know the color of a galaxy, we know how hot the stars are. From the galaxy's color we can find out how much light the stars in a galaxy are creating. Once we know how much light each star is creating, if we know how many total stars there are we can figure out how bright the galaxy should be at a certain distance.

\section{Color and Bumps Together Can Give Distance}

Imagine you are sitting close to a campfire. When you are right next to the fire, you can feel its warmth and you might even have enough light to read a book. But imagine that you start walking away from fire. 
Figure 2

Galaxy NGC 0524 is shown on the left. After a computer removes the main part of the galaxy from the image, only the bumps remain which is shown on the right. The sizes of the galaxy bumps depend on the galaxy's distance from us and its temperature. The picture of this galaxy was taken with the Hubble space telescope ${ }^{3}$.

\section{Figure 3}

This shows two campfires. The photo on the left ${ }^{4}$ is close enough that you can see different colors. If you were that close to the fire, you could feel its heat and even roast some marshmallows! On the right, the fire is still visible but dimmer, because it is farther away. The fire looks like it is just one color and you would not be able to feel its heat.

3 This website can tell you more about the Hubble:

http://hubblesite.org/

4 https://web.physics. wustl.edu/alford/ general/newton.html
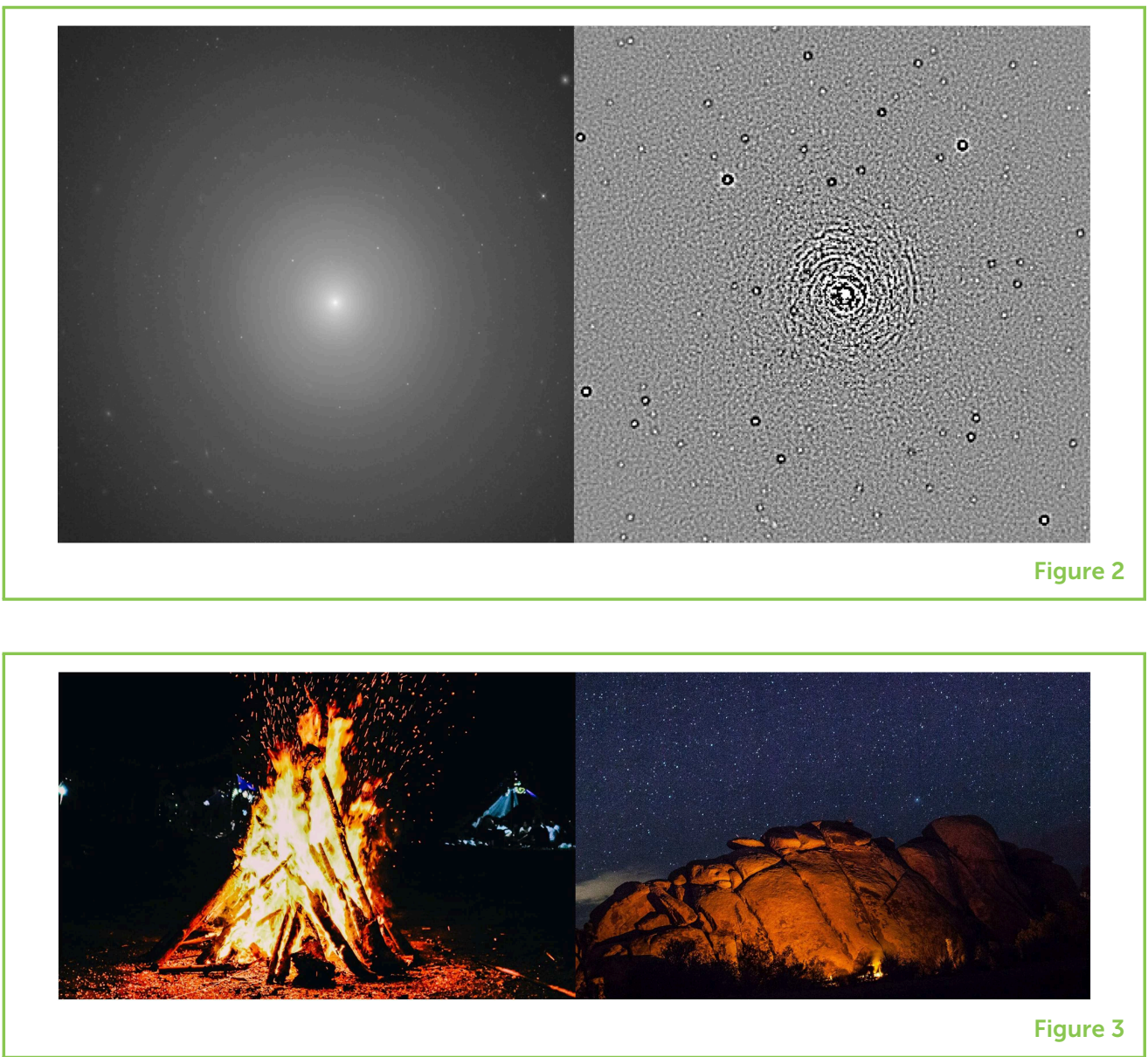

You will quickly feel colder and soon it will be too dark to read which you can see in Figure 3. The fire will also look like it is just one color, instead of a bunch of colors. That is not because the fire is going out, or because it is now a single color, but because you are farther away from the fire. If you carefully compared the way the fire looks when you are next to it with the way it looks from far away, you could calculate how far you walked. Just like with a campfire, we see less light and detail from galaxies the farther away they are.

A galaxy's bumpiness depends both on how far away it is and its color, which is why both kinds of data are needed. The color of a galaxy tells us how hot the stars are and how much light they produce. Once we know how hot the stars are and the size of the galaxy's bumps, we can figure out how many stars there are in that galaxy and how much light the galaxy is creating. From there, astronomers can finally calculate the distance the galaxy is from Earth, since they know how bright the galaxy should look if it were at a certain distance away, just like how you could measure how far you walked by comparing the light coming from the fire. 


\section{CALCULUS}

The super useful math Isaac Newton invented and used to do astronomy.

\section{WHY ARE DISTANCES IMPORTANT?}

There are a lot of reasons to measure distances to galaxies, even though it can be a lot of work. If we do not know the distance to a galaxy, we cannot figure out how big the galaxy is, we would not know how big the galaxy's black hole is, or how much stuff is in that galaxy, among so many things. It is very difficult to test other cool theories that astronomers have, such as theories of dark matter, dark energy, and other mysteries of the universe, if we do not know how far away things are! If we never figure out how to measure distances in space, we would not be able to understand what the universe is really like.

I have also been asked, "Why is it important to know what the universe is like?" It is important to understand how the universe works because, when we do, we can use that knowledge to create and do amazing things. Over 300 years ago, Sir Isaac Newton figured out how the planets orbit around the sun. That itself was a pretty cool discovery, but what most people do not realize is that, in making this discovery, he was also developing calculus ${ }^{4}$. Calculus is a type of math that has helped people invent things like satellites, computers, phones, the internet, and tools for doctors to save lives! Even the food you eat and the clothes you wear are there because scientists made cool discoveries about how our universe works.

So, we know that understanding distances in space is important, because this information will help us learn how the universe works. But why is SBF important to use? If you remember how big space is, and how everything is super far away, it is really difficult to measure the distances to far-away galaxies. We know how far away some galaxies are, but generally, the ones we know about are really close to Earth. If we want to measure the distance to a galaxy that is farther away, we can compare its color and bumpiness to those qualities of galaxies that are closer, and then make a distance measurement from those data. So, SBF can give us the distance to a galaxy that we otherwise would not have been able to measure.

\section{SO, WHAT IS NEXT?}

Because it takes a lot of work, SBF has only been performed on some galaxies. There will be many new telescopes in the future, taking lots of pictures, so we will have many more pictures then we have now. I am working on computer programs that will make it much faster to analyze these pictures and make distance measurements, so that astronomers can measure distances to as many galaxies as possible! 


\section{REFERENCES}

1. Adams, D. 1980. The Hitchhiker's Guide to the Galaxy. 1st Edn. New York, NY: Harmony Books.

SUBMITTED: 26 March 2019; ACCEPTED: 25 November 2019; PUBLISHED ONLINE: 12 December 2019.

EDITED BY: Joey Shapiro Key, University of Washington Bothell, United States

CITATION: Davis JD (2019) Measuring Distances to Galaxies. Front. Young Minds 7:142. doi: 10.3389/frym.2019.00142

CONFLICT OF INTEREST: The author declares that the research was conducted in the absence of any commercial or financial relationships that could be construed as a potential conflict of interest.

COPYRIGHT () 2019 Davis. This is an open-access article distributed under the terms of the Creative Commons Attribution License (CC BY). The use, distribution or reproduction in other forums is permitted, provided the original author(s) and the copyright owner(s) are credited and that the original publication in this journal is cited, in accordance with accepted academic practice. No use, distribution or reproduction is permitted which does not comply with these terms.

\section{YOUNG REVIEWERS}

\section{CAYLE, AGE: 13}

I am in seventh grade. In school I like writing, reading, math, and science. My favorite activities are softball, baking, running, and hanging out with my friends.

\section{EILEEN, AGE: 8}

Hi! My name is Eileen. I am 8 years old and just started third grade. I love drawing, singing, and making stuff. I want to be a chemist when I grow up. I am outgoing and I like to make friends. Regarding sports, I love rhythmic gymnastics, swimming, and jumping on the trampoline!

\section{AUTHOR}

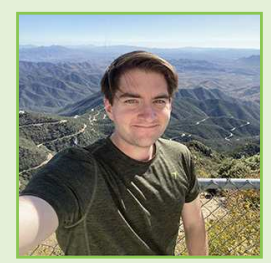

\section{JONATHAN D. DAVIS}

My name is Jonathan Davis. I am currently an observational astronomer for the University of Utah and the Smithsonian Astrophysical Observatory in Arizona, USA. I do really cool work like building software which finds stars we can take pictures of and measuring distances to galaxies! I also like to do other things like exercise and even play video games. If you are interested in some of the things I do in astronomy, you can check out my blog at: https://astronomaestro.blogspot.com. *astronomaestro@gmail.com 